\title{
Integrating Microsecond Circuit Switching into the Data Center
}

\author{
George Porter Richard Strong Nathan Farrington Alex Forencich Pang Chen-Sun \\ Tajana Rosing Yeshaiahu Fainman George Papen Amin Vahdat ${ }^{\dagger}$ \\ UC San Diego UC San Diego and Google, Inc. ${ }^{\dagger}$ \\ Text
}

\begin{abstract}
Recent proposals have employed optical circuit switching (OCS) to reduce the cost of data center networks. However, the relatively slow switching times (10-100 ms) assumed by these approaches, and the accompanying latencies of their control planes, has limited its use to only the largest data center networks with highly aggregated and constrained workloads. As faster switch technologies become available, designing a control plane capable of supporting them becomes a key challenge.

In this paper, we design and implement an OCS prototype capable of switching in $11.5 \mu \mathrm{s}$, and we use this prototype to expose a set of challenges that arise when supporting switching at microsecond time scales. In response, we propose a microsecond-latency control plane based on a circuit scheduling approach we call Traffic Matrix Scheduling (TMS) that proactively communicates circuit assignments to communicating entities so that circuit bandwidth can be used efficiently.
\end{abstract}

\section{Categories and Subject Descriptors}

C.2.1 [Computer-Communication Networks]: Network Architecture and Design_circuit-switching networks, packet-switching networks, network topology

\section{General Terms}

Design, Experimentation, Measurement, Performance

\section{Keywords}

Data Center Networks; Optical Networks

\section{INTRODUCTION}

As the size and complexity of data center deployments grow, meeting their requisite bisection bandwidth needs is a challenge. Servers with $10 \mathrm{Gbps}$ link rates are common today, and $40 \mathrm{Gbps}$ NICs are already commercially available. At large scale, this trend translates into significant bisection bandwidth requirements. For a large data center with numerous, rapidly changing applications,

Permission to make digital or hard copies of all or part of this work for personal or classroom use is granted without fee provided that copies are not made or distributed for profit or commercial advantage and that copies bear this notice and the full citation on the first page. Copyrights for components of this work owned by others than ACM must be honored. Abstracting with credit is permitted. To copy otherwise, or republish, to post on servers or to redistribute to lists, requires prior specific permission and/or a fee. Request permissions from permissions@acm.org.

SIGCOMM'13, August 12-16, 2013, Hong Kong, China.

Copyright 2013 ACM 978-1-4503-2056-6/13/08 ...\$15.00. supporting high bisection bandwidth is important, since ultimately application performance, and hence overall server utilization, may suffer if insufficient bandwidth is available. The result is that network complexity and expense are increasing.

To meet the required bandwidth demands, data center operators have adopted multi-layer network topologies [14] (e.g., folded Clos, or "FatTrees" $[1,16])$, shown in Figure 1(a). While these topologies scale to very high port counts, they are also a significant source of cost, due in part to the large amount of switches, optical transceivers, fibers, and power each of their layers requires. Recent efforts have proposed $[6,8,25]$ using optical circuit switches (OCS) to deliver reconfigurable bandwidth throughout the network, reducing some of the expense of multi-layer scale-out networks, shown in Figure 1(b). A key challenge to adopting these proposals has been their slow reconfiguration time, driven largely by existing 3D-MEMS technology limitations. Two components dominate this reconfiguration time: (1) the hardware switching time of the 3D-MEMS OCS (10-100 ms), and (2) the software/control plane overhead required to measure the communication patterns and calculate a new schedule (100ms to $1 \mathrm{~s}$ ). As a result, the control plane is limited to supporting only highly aggregated traffic at the core of the network [8], or applications constrained to have high traffic stability [25].

As optical switches become faster, deploying them more widely in data center networks (e.g., to interconnect top-of-rack (ToR) switches) requires a correspondingly faster control plane capable of efficiently utilizing short-lived circuits. The contribution of this paper is such a control plane. To gain experience with fast OCS switching, we start by designing and building a simple 24-port OCS prototype called Mordia, ${ }^{1}$ which has a switch time of $11.5 \mu \mathrm{s}$. Mordia is built entirely with commercially available components, most notably 2D-based MEMS wavelength-selective switches (WSS). We use this prototype as a stand-in for future low-latency OCS devices.

Using the Mordia prototype as a starting point, we then identify a set of challenges involved in adopting existing OCS control planes to microsecond-latency switching. In response to these challenges, we propose a new circuit scheduling approach called Traffic Matrix Scheduling (TMS). Instead of measuring long-lived, prevailing conditions and configuring the topology in response, TMS instead leverages application information and short-term demand estimates to compute short-term circuit schedules. TMS chooses these schedules to rapidly multiplex circuits across a set of end points, making use of the fast circuit switch time to reduce buffering and network delay. To obtain high circuit utilization, the computed schedules

\footnotetext{
${ }^{1}$ Microsecond Optical Research Data Center Interconnect Architecture
} 


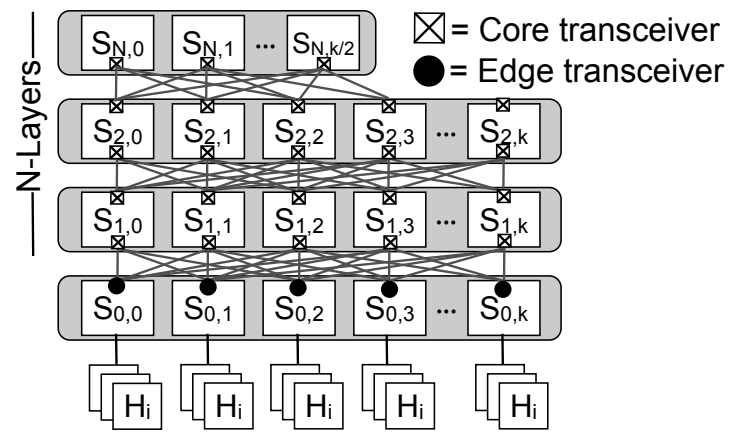

(a) A FatTree network topology

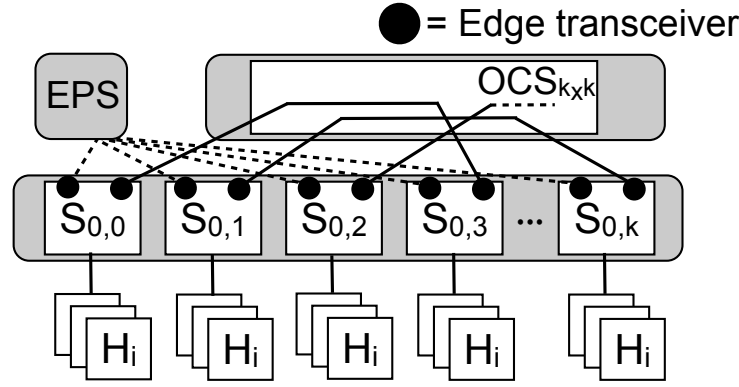

(b) A Hybrid network topology

Figure 1: A comparison of a scale-out, multi-layered FatTree network and a Hybrid electrical/optical network design. In the FatTree topology (a) each layer of switching incurs additional cost in electronics, core transceivers, fiber cabling, and power. In contrast, the Hybrid topology (b) requires only a single "layer" assuming that the OCS reconfiguration speed is sufficiently fast.

\begin{tabular}{ccccc}
\hline Speed & Radix & Depth & $\begin{array}{c}\text { \# Nodes } \\
\text { (x1000) }\end{array}$ & $\begin{array}{c}\text { \# Core Ports } \\
\text { (x1000) }\end{array}$ \\
\hline \multirow{2}{*}{$10 \mathrm{G}$} & 48 & 5 & 498 & 3,484 \\
\cline { 2 - 5 } & 96 & 3 & 28 & 83 \\
\hline \multirow{3}{*}{$40 \mathrm{G}$} & \multirow{2}{*}{16} & 7 & 33 & 360 \\
\cline { 2 - 5 } & & 9 & 524 & 7,864 \\
\hline & \multirow{2}{*}{24} & 5 & 16 & 109 \\
\cline { 3 - 5 } & & 7 & 560 & 6,159 \\
\hline
\end{tabular}

Table 1: The complexity of sample multi-layer, fullyprovisioned, scale-out network topologies. Small-radix switches and link redundancy require more layers, and thus more switches and optical transceivers, driving up their cost.

are communicated to ToRs connected to Mordia, which adjust the transmission of packets into the network to coincide with the scheduled switch reconfigurations, with full knowledge of when bandwidth will be most available to a particular destination. In this way, both short and long flows can be offloaded into the OCS.

As a result, TMS can achieve $65 \%$ of the bandwidth of an identical link rate electronic packet switch (EPS) with circuits as short as $61 \mu$ s duration, and $95 \%$ of EPS performance with $300-\mu$ s circuits using commodity hardware. Taken together, our work suggests that continuing to push down the reconfiguration time of optical switches and reducing the software and control overheads holds the potential to radically lower the cost for delivering high bisection bandwidth in the data center.

\section{MOTIVATION: REDUCING NETWORK COST VIA FASTER SWITCHING}

We now examine some of the sources of data center costs, and motivate the need for low-latency circuit switching.

\subsection{Multi-layer switching networks}

Multi-layer switching topologies like FatTrees are highly scalable and flexible - any node can communicate with any other node on demand. However, they must be provisioned for worstcase communication patterns, which can require as many as five to nine layers in the largest networks, with each subsequent layer less utilized than the next in the common case. Each of these lay- ers adds substantial cost in terms of the switch hardware, optical transceivers, fibers, and power.

Consider an $N$-level scale-out FatTree data center network supporting $M$ servers partitioned into racks (e.g., 20 to 40 servers per rack). Such a network built from $k$-radix switches can support $k^{N} / 2^{N-1}$ servers, with each layer of switching requiring $k^{N-1} / 2^{N-2}$ switches (though layer $N$ itself requires half this amount). The choice of the number of layers in the network is determined by the number of hosts and the radix $k$ of each switch. Given a particular data center, it is straightforward to determine the number of layers needed to interconnect each of the servers.

There are two trends that impact the cost of the network by increasing the number of necessary layers of switching: fault tolerance and high link rates. We consider each in turn.

Fault tolerance: While a FatTree network can survive link failures by relying on its multi-path topology, doing so incurs a networkwide reconvergence. This can be highly disruptive at large scale, and so redundant links are used to survive such failures [24]. Dual link redundancy, for instance, effectively cuts the radix of the switch in half since each logical link now requires two switch ports.

High link rates: For relatively mature link technologies like 10 Gbps Ethernet, high-radix switches are widely available commercially: 10 Gbps switches with 64 or even 96 ports are becoming commodity. In contrast, newer generations of switches based on 40 Gbps have much lower radices, for example 16 to 24 ports per switch. Hence, as data center operators build out new networks based on increasingly faster link rates, it will not always be possible to use high radix switches as the fundamental building block.

These constraints necessitate additional switching layers and, thus, additional cost and complexity. Table 1 shows the number of core network ports (ports used to connect one layer of switching to an adjacent layer) for a set of data center sizes and switch radices. Note that since this table shows fully-provisioned topologies, it serves as an upper bound to what might be built in practice since the network might be only partially provisioned depending on the number of nodes that need to be supported.

\subsection{OCS model}

We now describe a simple model of an OCS suitable for interconnecting ToR switches. This model is similar to that assumed by previous hybrid network designs $[6,8,25]$, but with a key difference: orders of magnitude faster switching speed.

The model consists of an N-port optical circuit switch with a reconfiguration latency of $O(10) \mu$ s. Each input port can be mapped 
to any output port, and these mappings can be changed arbitrarily (with the constraint that only one input port can map to any given output port). The OCS does not buffer packets, and indeed does not interpret the bits in packets at all — the mapping of input ports to output ports is entirely controlled by an external scheduler. This scheduler is responsible for determining the time-varying mapping of input ports to output ports and programming the switch accordingly.

We assume that ToRs attached to the OCS support per-destination flow control, meaning that packets for destination $D$ are only transmitted to an OCS input port when a circuit is setup between that input port and $D$. Packets to destinations other than $D$ are queued in the edge ToR during this time. Furthermore, during the OCS reconfiguration period, all packets are queued in the ToR. Since the OCS cannot buffer packets, the ToR must be synchronized to only transmit packets at the appropriate times. This queueing can lead to significant delay, especially for small flows that are particularly sensitive to the observed round-trip time. In these cases, packets can be sent to a packet switch in the spirit of other hybrid network proposals. In this work, we focus on the OCS and its control plane in isolation, concentrating particularly on reducing end-to-end reconfiguration latency. In this way, our work is complementary to other work in designing hybrid networks.

\section{MICROSECOND SCHEDULING}

A key challenge in supporting microsecond-latency OCS switches is effectively making use of short-lived circuits. In [7], we proposed an approach for scheduling circuits, called Traffic Matrix Scheduling (TMS). In this section, we expand on that initial idea, and then implement and evaluate it in the context of a testbed deployment later in the paper. For now, we assume that the network-wide traffic demand is known and return to the issue of estimating demand at the end of the section.

\subsection{Overview}

Existing approaches that integrate OCS hardware into the data center amortize the long switching time (tens of milliseconds) of previous generation optical technology by reconfiguring the OCS only once every few 100s of milliseconds or even several seconds. The substantial interval between reconfigurations affords their underlying control loops the opportunity to estimate demand, calculate an optimal OCS configuration, and communicate it across the network every time the switch is repositioned.

Previous hybrid networks perform what we call hotspot scheduling (HSS). HSS (a) measures the inter-rack traffic matrix, (b) estimates the traffic demand matrix, (c) identifies hotspots, and (d) uses a centralized scheduler to establish physical circuits between racks to offload only the identified hotspot traffic onto the circuitswitched network. The remaining traffic is routed over the packetswitched network. Because of the substantial delay between reconfigurations, HSS can employ complex methods and algorithms to estimate demand and identify hotspots. Errors in identifying hotspots, however, can lead to significant losses in efficiency. If a selected hotspot does not generate sufficient traffic to saturate a circuit, then the remaining capacity goes to waste for the (non-trivial) duration of the current configuration.

When the OCS can be reconfigured on the order of $10 \mathrm{~s}$ of $\mu \mathrm{s}$, we argue that it is possible to route most of the traffic over circuits. In contrast to HSS, we propose an approach called "Traffic Matrix Switching" (TMS) that estimates demand and calculates a short-term schedule that moves the OCS rapidly through a sequence of configurations to service predicted demand. By rapidly time-sharing circuits across many destinations at microsecond time

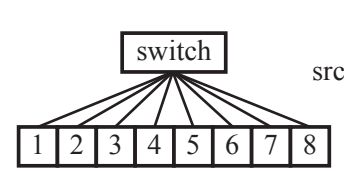

(a)

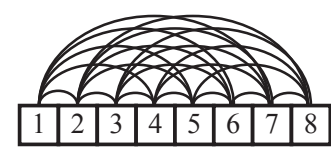

(b)

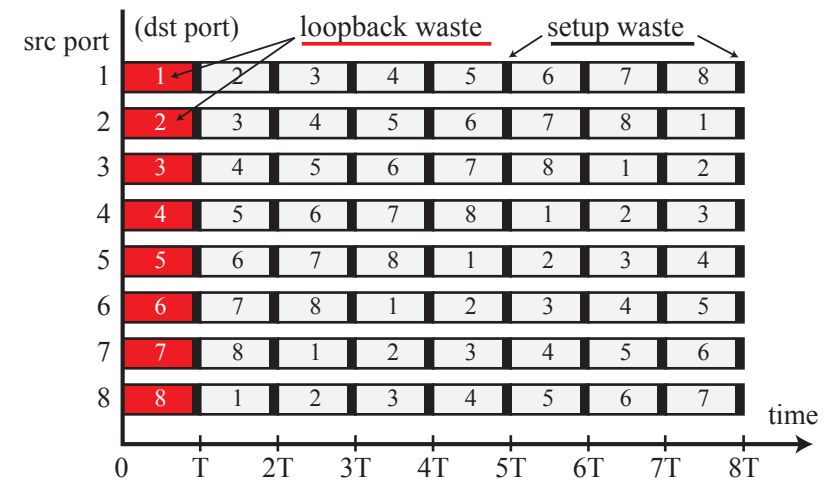

(d)

Figure 2: Eight racks running Hadoop with an all-to-all communication pattern: (a) physical topology, (b) logical topology, (c) inter-rack traffic demand matrix, (d) circuit switch schedule.

scales, TMS is able to make more effective use of the circuit bandwidth (and reduces to hotspot scheduling when demand is extremely concentrated). The key insight is that by sending the upcoming schedule to both the OCS and the ToRs, they can effectively make use of each circuit as it becomes available. Moreover, while the current schedule is being carried out, the control loop can enter its next iteration. In this way, the running time of the control plane is decoupled from the switch speed. In particular, the control plane only needs to recompute schedules fast enough to keep up with shifts in the underlying traffic patterns, rather than with the OCS switch speed.

One of the key reasons that we adopted TMS instead of running a hotspot scheduling algorithm faster is that hotspot scheduling is inherently stateless. Each iteration of hotspot scheduling greedily chooses a perfect matching of circuit assignments to find the largest elephants ( $k$ for a $k$-by- $k$ switch). This doesn't necessarily result in ideal usage of the OCS. Further, the control plane would then have to run at the speed of the underlying host burst behavior. With TMS, we can compute an entire "week" of schedules once, amortizing the control loop time over a number of switch reconfigurations. We believe that this is critical to match the speed of the OCS.

\subsection{Example}

Consider eight racks running Hadoop and generating a perfectly uniform all-to-all communication pattern. Figure 2(a) shows the racks physically connected to the same core-circuit switch; Fig- 
Step 1. Gather traffic matrix M

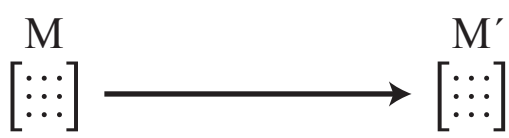

Step 3. Decompose $\mathrm{M}^{\prime}$ into schedule

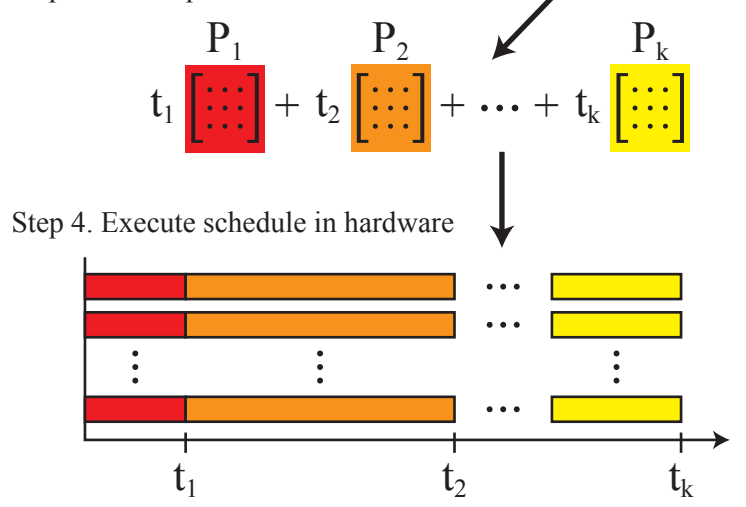

Figure 3: Steps of the traffic matrix scheduling algorithm.

ure 2(b) shows the logical connectivity, and Figure 2(c) shows the inter-rack traffic demand matrix with sources as rows, destinations as columns, and values as fractions of the total link rate. The diagonal is not zero because hosts send to other hosts in the same rack. Although this intra-rack traffic does not transit the core circuit switch, it is still accounted for in the traffic demand matrix. This matrix is the desired transmission rate of the hosts, and it is the responsibility of the network to satisfy this demand.

The Gantt chart in Figure 2(d) shows a circuit switch schedule that partitions time into eight equal-duration time slots. Over the course of the schedule, each source port will connect to each destination port for exactly $1 / 8$ of the total time. It thus implements the logical full mesh topology in Figure 2(b) and allows all of the traffic to be routed. The schedule then repeats.

A circuit switch schedule, however, has two sources of waste. First, loopback traffic does not leave the rack and transit the circuit switch, so any circuit switch loopback assignments are wasted, such as the assignment from $t=0$ to $t=T$. Second, the circuit switch takes a non-negligible amount of time to switch and setup new circuits ( $\left.t_{\text {setup }}\right)$, which we represent as black bars at the end of each time slot. No traffic can transit the circuit switch during this time. Reducing loopback waste requires careful scheduling, whereas reducing setup waste requires faster switching. Finally, note that although this example produces a repeating schedule, TMS can generate arbitrary time-varying circuit assignments as we describe below.

\subsection{Schedule computation}

The TMS algorithm is divided into a set of steps, as shown in Figure 3. First, the traffic demand matrix (TDM) is scaled into a bandwidth allocation matrix (BAM). A TDM represents the amount of traffic, in units of circuit line rate, that the hosts in a source rack wish to transmit to the hosts in a destination rack. A BAM, on the other hand, represents the fraction of circuit bandwidth the switch should allocate between each input-output port pair in an ideal schedule. In general, the TDM may not be admissible (i.e., the total demand is greater than the network capacity). In practice, though, the network is rarely driven to full utilization, so we need to scale "up" the TDM to arrive at a BAM. If no rack wishes to send more than its link rate (its row sum is less than or equal to 1) and no rack wishes to receive more than its link rate (its column sum is less than or equal to 1), then we say that the TDM is both admissible and doubly substochastic. The goal of scaling the TDM is to compute a doubly stochastic BDM where its row sums and column sums are all exactly equal to $1-$ meaning the circuits would be fully utilized. By scaling the TDM into a BAM, we simultaneously preserve the relative demands of the senders and receivers while satisfying the constraints of the circuit switch. Several matrix scaling algorithms can be used for this purpose. Sinkhorn's algorithm [18] is particularly attractive because it works even when the originally TDM is not admissible (i.e., the network is over driven).

Next, the BAM is decomposed into a circuit switch schedule, which is a convex combination of permutation matrices that sum to the original BAM,

$$
\mathrm{BAM}=\sum_{i}^{k} c_{i} P_{i}
$$

where $0 \leq i \leq k$, and $k=N^{2}-2 N+2$. Each permutation matrix, $P_{i}$, represents a circuit switch assignment, and each scalar coefficient, $c_{i}$, represents a time slot duration as a fraction of the total schedule duration. A variety of matrix decomposition algorithms exist. We employ an algorithm originally due to Birkhoff-von Neumann $(\mathrm{BvN})[5,23]$ that can decompose any doubly stochastic matrix, implying we can always compute a perfect schedule given a BAM. Improved versions of the classic $\mathrm{BvN}$ algorithm have running times between $\mathrm{O}\left(n \log ^{2} n\right)$ and $\mathrm{O}\left(n^{2}\right)$ [11].

\subsection{Demand estimation}

Traffic matrix scheduling, just like hotspot scheduling, requires an estimate of the network-wide demand. There are several potential sources of this information. First, packet counters in the ToRs can be polled to determine the traffic matrix, and from that the demand matrix can be computed using techniques presented in Hedera [2]. This method would likely introduce significant delays, given the latency of polling and running the demand estimator. A second potential approach, if the network is centrally controlled, is to rely on OpenFlow [15] network controllers to provide a snapshot of the overall traffic demand. Third, an approach similar to that taken by c-Through [25] may be adopted: A central controller, or even each ToR, can query individual end hosts and retrieve the TCP send buffer sizes of active connections. Asynchronously sending this information to the ToRs can further reduce the latency of collecting the measurements. Finally, application controllers, such as the Hadoop JobTracker [12], can provide hints as to future demands. Our prototype implementation does not implement demand estimation.

\section{ANALYSIS}

The throughput of a network that uses circuit switching is constrained by the network's duty cycle, and its feasibility is constrained by the amount of buffering required and the circuit schedule. We consider these issues in turn.

\subsection{Duty cycle and effective link rate}

In a circuit-switched network, there is a finite reconfiguration time, or setup time $t_{\text {setup }}$, during which no data can be sent. If the link data rate is $R_{\text {link }}$, then the effective data rate $R$ of each circuit is $R=D R_{\text {link }}$, where:

$$
D=\frac{t_{\text {stable }}}{t_{\text {setup }}+t_{\text {stable }}}
$$

is the duty cycle and $t_{\text {stable }}$ is the time that the circuit is "open" and can carry traffic. For example, if $R_{\text {link }}$ is $10 \mathrm{Gbps}$ and $D$ is $90 \%$, 


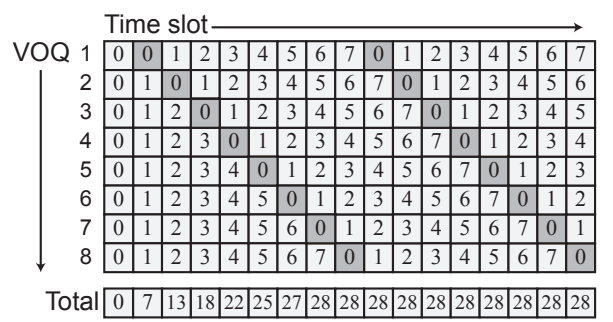

Figure 4: Virtual output queue (VOQ) buffer occupancies for a ToR from cold start.

which is representative of the Mordia OCS, then the effective link rate $R$ is $9 \mathrm{Gbps}$.

The duty cycle can be increased by reducing the setup time or increasing the duration that a circuit is open. Reducing setup time $t_{\text {setup }}$ depends on switch technology. The duration $t_{\text {stable }}$ that a circuit is open is controllable. However, having circuits that are open for a long period of time affects the amount of buffering that is required at the host, as we discuss below. Since the Mordia OCS is rate agnostic, it is also possible to increase overall delivered bandwidth by using faster optical transceivers to increase the link rate while simultaneously reducing the duty cycle for the circuitswitched portion of the network.

\subsection{Buffer requirements}

Buffering is required at the source ToR in a circuit-switched network because there is not always a circuit established between a particular source and destination. In this section, we analyze these buffering requirements.

Assume that each ToR connected to the Mordia switch maintains a set of $N$ virtual output queues (VOQs) [20], one for every possible circuit destination. Strict VOQ is not required, but the ToR must maintain at least one set of queues for each possible destination. When a circuit is established, all traffic destined for that particular destination is drained from the respective queue. Figure 4 shows the buffer occupancies of these VOQs of a ToR from a cold start, in units of the slot time $T$ (without loss of generality, we assume uniform slot times here). In less than one complete scheduling period a ToR has filled its VOQs to the steady-state level. A particular queue fills at a rate dictated by the traffic matrix until a circuit is established to the appropriate destination. The queue is drained over the duration that the circuit is open.

For an all-to-all workload with $N$ ToRs, an effective link rate of $R$ bits per second, and a slot duration of $T$ seconds, the buffering required by each host is:

$$
B=R(N-1) T \quad \text { (bits) }
$$

Examining (3) shows why millisecond switching times are simply too large to support traffic matrix scheduling. For example if $R$ $=9 \mathrm{Gbps}, N=64$, and $T=100 \mathrm{~ms}$, then $B$ is $7.1 \mathrm{~GB}$ of buffering per OCS port, which is not currently practical. Given that $R$ and $N$ are both likely to increase in future data centers, the only way to make traffic matrix scheduling practical is to decrease the slot time $T$ by using, e.g., microsecond switching. Setting $T=100 \mu$ s yields $B=7.1 \mathrm{MB}$ of buffering per port, which is currently practical. Reducing buffering within the ToR leads to more practical and lower cost designs.

\subsection{Longest time-slot first scheduling}

While BvN always produces a schedule that eventually serves all input-output pairs according to their demand, sometimes it may

\begin{tabular}{rrrr}
\hline$n$ & Circuit & Packet & D \\
\hline 0 & $0 \%$ & $100.0 \%$ & N/A \\
1 & $39.4 \%$ & $60.6 \%$ & $100.0 \%$ \\
2 & $53.8 \%$ & $46.2 \%$ & $98.0 \%$ \\
3 & $63.8 \%$ & $36.2 \%$ & $97.0 \%$ \\
4 & $72.7 \%$ & $27.3 \%$ & $96.0 \%$ \\
5 & $80.6 \%$ & $19.4 \%$ & $95.0 \%$ \\
6 & $87.3 \%$ & $12.7 \%$ & $94.0 \%$ \\
7 & $92.3 \%$ & $7.7 \%$ & $93.0 \%$ \\
8 & $96.6 \%$ & $3.4 \%$ & $92.0 \%$ \\
9 & $99.3 \%$ & $0.7 \%$ & $91.0 \%$ \\
10 & $100.0 \%$ & $0 \%$ & $90.0 \%$ \\
\hline
\end{tabular}

Table 2: Example of the tradeoff between the number of schedule time slots $(n)$, the amount of traffic sent over the opticalcircuit switched network (Circuit) vs. the packet-switched network (Packet), and the duty cycle $(D)$ for a randomly generated TDM with a reconfiguration latency of $10 \mu \mathrm{s}$.

be better not to schedule all traffic over the circuit switch and to simply schedule only the longest time slots. The reason is that the BvN decomposition algorithm generates time slots of different lengths, some of which can be quite short (e.g., less than $1 \%$ of the entire schedule). With such a short time slot, it is likely that the OCS switching time would dominate any bandwidth delivered during those small slots. In these cases, it is better to route that traffic over the packet-switched network.

The greatest benefit comes from scheduling only the first $n$ time slots, where $n$ is chosen based on both the minimum required duty cycle, $D$, as well as the maximum allowed schedule length. Any remaining traffic is instead sent to the packet-switched network. Using the same randomly generated TDM from [7], we calculate the tradeoffs in different numbers of slots for the schedule, as shown in Table 2 .

As $n$ increases, an increasing fraction of the total traffic transits the circuit-switched network. In the limit when $n=k$, all traffic is sent over the circuit-switched network. However, the duty cycle decreases with increasing $n$, since the schedule invokes more switch reconfigurations. For example, if the minimum required duty cycle was $95 \%$, then by setting $n=5,80.6 \%$ of the total traffic would be routed over circuit switches. Alternatively, at the cost of additional host buffering, we could increase $n$ to 6 or 7 while keeping the duty cycle at $95 \%$.

\section{IMPLEMENTATION}

We evaluated the Mordia OCS in a testbed environment. This implementation effort consists of two primary tasks: (1) selecting an OCS capable of switching at $\mathrm{O}(10) \mu \mathrm{s}$, and (2) modifying ToRs to support flow control on a per-destination basis at microsecond timescales. Unfortunately, as we discuss in Section 8, the only practical commercially available OCSes that can switch in sub$100 \mu$ s timescales have small port counts (e.g., 4 or 8 ports). To evaluate at the scale of a ToR (e.g., 24-48 hosts), we instead build our own prototype OCS supporting 24 ports based on commercial wavelength-selective switches, described in Section 5.1. Instead of building our own ToRs with our flow control requirements, we instead emulate them using commodity Linux servers, as described in Section 5.2. 


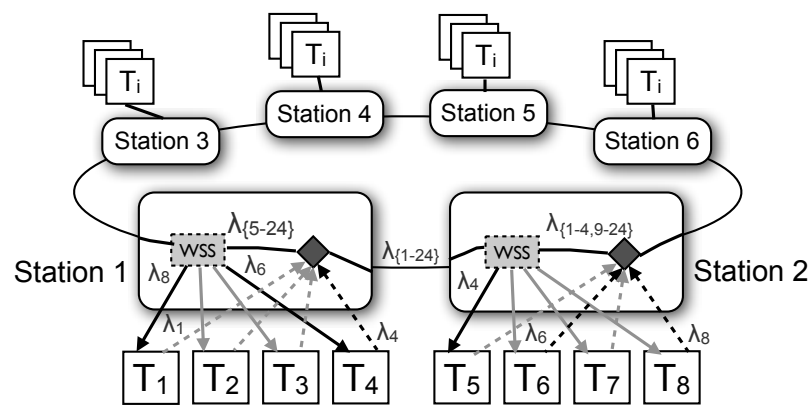

Figure 5: The Mordia OCS prototype, which consists of a ring conveying $N$ wavelengths through six stations. Each source ToR transmits on its own wavelength, and each station forwards a subset of four wavelengths to the ToRs attached to it. This prototype supports an arbitrary reconfigurable mapping of source to destination ports with a switch time of $11.5 \mu \mathrm{s}$.

\subsection{Mordia prototype}

The Mordia prototype is a 24-port OCS that supports arbitrary reconfiguration of the input-to-output port mappings. We first describe the underlying technology we leveraged in building the OCS, then describe its design.

\subsubsection{Technology}

Unlike previous data center OCS designs [8,25], we chose not to use 3D-MEMS based switching due to its high reconfiguration time. The maximum achievable speed of a 3D-MEMS space switch depends on the number of ports, since more ports require precise analog control of the 2-axis orientation of relatively large mirrors. Since the mirror response time depends on the size and angular range, there is in general a design tradeoff between the switch port count, insertion loss, and switching speed. As a result, commercial 3D-MEMS switches support reconfiguration times in the 10s of milliseconds range [10].

Another type of optical circuit switch is a wavelength-selective switch (WSS). It takes as input a fiber with $N$ wavelengths in it, and it can be configured to carry any subset of those $N$ wavelengths to $M$ output ports. Typically a WSS switch has an extra "bypass" port that carries the remaining $N-M$ frequencies. We call this type of WSS switch a $1 \times M$ switch, and in our prototype, $M=4$. Our switch does not have a bypass port, and so we implement the bypass functionality external to the WSS using additional optical components.

The internal switching elements used in a wavelength-selective switch can be built using liquid crystal technology or MEMS [9]. Most MEMS WSSes use analog tilt to address multiple outputs, but at least one commercial WSS has been built using binary MEMSbased switches [19]. Binary MEMS switching technology uses only two positions for each mirror moving between two mechanically stopped angles, and also uses much smaller mirrors with respect to a 3D-MEMS space switch. A similar binary MEMS switch is used for commercial projection televisions. The binary switching of small mirror elements results in an achievable switching speed that is several orders of magnitude faster than a commercial 3DMEMS switch.

In general, there is a tradeoff between 3D-MEMS, which offers high port count at relatively slow reconfiguration time, and 2DMEMS, which offers microsecond switching time at small port counts (e.g., $1 \times 4$ or $1 \times 8$ ). The key idea in the Mordia OCS prototype is to harness six $1 \times 4$ switches with bypass ports to build a single $24 \times 24$-port switch. We now briefly summarize the operation of the data path.

\subsubsection{Data plane}

The Mordia OCS prototype is physically constructed as a unidirectional ring of $N=24$ individual wavelengths carried in a single optical fiber. Each wavelength is an individual channel connecting an input port to an output port, and each input port is assigned its own specific wavelength that is not used by any other input port. An output port can tune to receive any of the wavelengths in the ring, and deliver packets from any of the input ports. Consequently, this architecture supports circuit unicast, circuit multicast, circuit broadcast, and also circuit loopback, in which traffic from each port transits the entire ring before returning back to the source. We note that although the data plane is physically a ring, any host can send to any other host, and the input-to-output mapping can be configured arbitrarily (an example of which is shown in Figure 5).

Wavelengths are dropped and added from/to the ring at six stations. A station is an interconnection point for ToRs to receive and transmit packets from/to the Mordia prototype. To receive packets, the input containing all $N$ wavelengths enters the WSS to be wavelength multiplexed. The WSS selects four of these wavelengths, and routes one of each to the four WSS output ports, and onto the four ToRs at that station. To transmit packets, each station adds four wavelengths to the ring, identical to the four wavelengths the station initially drops. To enable this scheme, each station contains a commercial $1 \times 4$-port WSS.

\subsubsection{ToRs}

Each ToR connects to the OCS via one or more optical uplinks, and internally maintains $N-1$ queues of outgoing packets, one for each of the $N-1$ OCS output ports. The ToR participates in a control plane, which informs each ToR of the short-term schedule of impending circuit configurations. In this way, the ToRs know which circuits will be established in the near future, and can use that foreknowledge to make efficient use of circuits once they are established.

Initially, the ToR does not send any packets into the network, and simply waits to become synchronized with the Mordia OCS. This synchronization is necessary since the OCS cannot buffer any packets, and so the ToR must drain packets from the appropriate queue in sync with the OCS's circuit establishment. Synchronization consists of two steps: (1) receiving a schedule from the scheduler via an out-of-band channel (e.g., an Ethernet-based management port on the ToR), and (2) determining the current state of the OCS. Step 2 can be accomplished by having the ToR monitor the link up and down events and matching their timings with the schedule received in Step 1. Given the duration of circuit reconfiguration is always $11.5 \mu \mathrm{s}$, the scheduler can artificially extend one reconfiguration delay periodically to serve as a synchronization point. The delay must exceed the error of its measurement and any variation in reconfiguration times to be detectable (i.e., must be greater than $1 \mu \mathrm{s}$ in our case). Adding this extra delay incurs negligible overhead since it is done infrequently (e.g., every second).

We use the terminology day to refer to a period when a circuit is established and packets can transit a circuit, and we say that night is when the switch is being reconfigured, and no light (and hence no packets) are transiting the circuit. The length of a single schedule is called a week, and the day and week lengths can vary from day-to-day and from week-to-week. When the OCS is undergoing reconfiguration, each ToR port detects a link down event, and night begins. Once the reconfiguration is complete, the link comes back up and the next "day" begins. 
During normal-time operation, any data received by the ToR from its connected hosts is simply buffered internally into the appropriate queue based on the destination. The mapping of the packet destination and the queue number is topology-specific, and is configured out-of-band via the control plane at initialization time and whenever the topology changes. When the ToR detects that day $i$ has started, it begins draining packets from queue $i$ into the OCS. When it detects night time (link down), it re-buffers the packet it was transmitting (since that packet likely was truncated midtransmission), and stops sending any packets into the network.

\subsubsection{Data plane example}

Figure 5 shows an overview of the Mordia prototype's data path. In this example, there are three circuits established: one from $T_{6}$ to $T_{4}$, one from $T_{8}$ to $T_{1}$, and one from $T_{4}$ to $T_{5}$. Consider the circuit from $T_{4}$ to $T_{5} . T_{4}$ has a transceiver with its own frequency, shown in the Figure as $\lambda_{4}$. This signal is introduced into the ring by an optical mux, shown as a black diamond, and transits to the next station, along with the other $N-1$ frequencies. The WSS switch in Station 2 is configured to forward $\lambda_{4}$ to its first output port, which corresponds to $T_{5}$. In this way, the signal from $T_{4}$ terminates at $T_{5}$. The $N-4$ signals that the WSS is not configured to map to local ToRs bypass the WSS, which is shown as $\lambda_{\{1-4,9-24\}}$. These are re-integrated with the signals from ToRs $T_{5}$ through $T_{8}$ originating in Station 2, and sent back into the ring. A lower-bound on the end-to-end reconfiguration time of such a network is gated on the switching speed of the individual WSS switches, which we evaluate in Section 6.1.

\subsubsection{Implementation details}

The implementation of the hardware for the Mordia prototype consists of four rack-mounted sliding trays. Three of these trays contain the components for the six stations with each tray housing the components for two stations. The fourth tray contains power supplies and an FPGA control board that implements the scheduler. This board is based on a Xilinx Spartan-6 XC6SLX45 FPGA device. Each tray contains two wavelength-selective switches, which are $1 \times 4$ Nistica Full Fledge 100 switches. Although these switches can be programmed arbitrarily, the signaling path to do so has not yet been optimized for low latency. Thus we asked the vendor to modify the WSS switches to enable low-latency operation by supporting a single signaling pin to step the switch forward through a programmable schedule. As a result, although our prototype only supports weighted round-robin schedules, those schedules can be reprogrammed on a week-to-week basis. This limitation is not fundamental, but rather one of engineering expediency.

\subsection{Emulating ToRs with commodity servers}

To construct our prototype, we use commodity servers to emulate each of the ToRs. Although the Mordia OCS supports 24 ports, our transceiver vendor was not able to meet specifications on one of those transceivers, leaving us with 23 usable ports in total. Each of our 23 servers is an HP DL 380G6 with two Intel E5520 4-core CPUs, 24 GB of memory, and a dual-port Myricom 10G-PCIE-8B 10 Gbps NIC. One port on each server contains a DWDM 10 Gbps transceiver, taken from the following ITU-T DWDM laser channels: $15-18,23-26,31-34,39-42,47-50$, and 55-58. Each server runs Linux 2.6.32.

\subsubsection{Explicit synchronization and control}

Each of the emulated ToRs must transmit packets from the appropriate queue in sync with the OCS with microsecond precision. The source code to our NIC firmware is not publicly available,

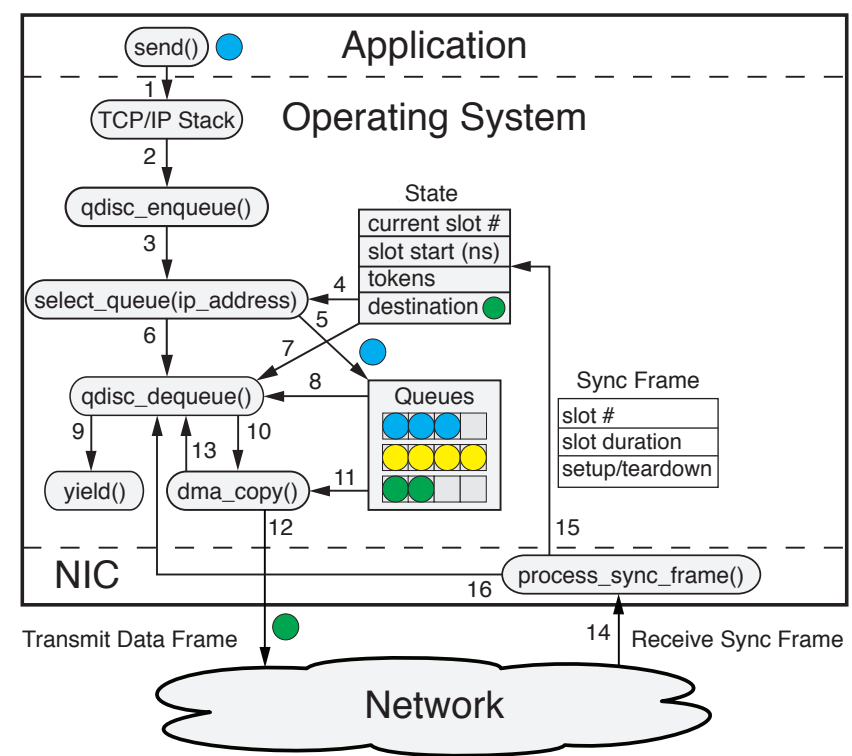

Figure 6: A software implementation of multi-queue support in Linux using commodity Ethernet NICs. Sync frames coordinate state between each emulated ToR (server) and the scheduler, so that each Qdisc knows when to transmit Ethernet frames.

and so we cannot detect link up and down events in real time and cannot implement the synchronization approach presented in Section 5.1.3. Instead, we have modified our prototype to include a separate synchronization channel between the scheduler and the servers that the scheduler uses to notify the servers when the switch is being reconfigured. Ethernet NICs do not typically provide much direct control over the scheduling of packet transmissions. Thus we have implemented a Linux kernel module to carry out these tasks. We now describe how we modified the Linux networking stack on each server to support circuit scheduling, and to remain synchronized with the OCS.

We modify the OS in three key ways. First, we adapt the Ethernet NIC driver to listen for synchronization packets from the scheduler so that the host knows the current state of the OCS. Second, we modify the NIC driver to ignore the "link-down" events that occur when the OCS is reconfiguring. Third, we add a custom queuing discipline (Qdisc) that drains packets from queues based on the configuration of the OCS.

Synchronization packets: The OCS FPGA controller transmits synchronization packets to a separate 10G packet-switched network which connects to a second Ethernet port on each server. These packets are sent before and after reconfiguration so that all connected devices know the state of the OCS. The packets include the slot number, the slot duration, and whether the circuits are being setup or torn down. The connected devices maintain a map between the slot number and each destination. The Ethernet NIC driver also maintains a data structure with a set of per-circuit tokens to control the data transmission time and rate. Note that this separate signaling network would not be needed in a production system where access to the NIC firmware would be available.

Link-down events: Since the OCS is switching rapidly, the host NIC ports attached to the OCS experience numerous link-up and link-down events. When Linux receives a link-down event, it normally disables the interface and resets and closes any open sockets 
and TCP connections. To prevent these resets, we disable the linkdown and link-up calls in the NIC driver.

Mordia Qdisc (shown in Figure 6): When a user's application sends data, that data transits the TCP/IP stack (1) and is encapsulated into a sequence of Ethernet frames. The kernel enqueues these frames into our custom Qdisc (2), which then selects (3) one of multiple virtual output queues (VOQs) based on the packet's IP address and the queue-to-destination map (4). The Ethernet frame is enqueued (5) and the qdisc_dequeue function is scheduled (6) using a softirq. The qdisc_dequeue function reads the current communication slot number (7) and checks the queue length (8). If there is no frame to transmit, control is yielded back to the OS (9). If there is a frame to transmit, the frame is DMA copied to the Ethernet NIC (10-12). The total number of packets sent directly corresponds to the number of tokens accumulated in the Ethernet NIC's data structure to control the timing and the rate. The kernel then schedules the qdisc_dequeue function again (13) until VOQ is empty and control is yielded back to the OS (9). When the next sync frame arrives from the controller (14), it is processed, and the scheduling state is updated (15). Then the kernel schedules the qdisc_dequeue function with a softirq in case there are frames enqueued that can now be transmitted (16). Given that all the packets are only transmitted during the times that the slot is active, the code for receiving packets did not need to be modified.

\section{EVALUATION}

Our evaluation seeks to:

1. Determine the baseline end-to-end reconfiguration time of the Mordia OCS as seen by ToRs. We find that, accounting for the hardware and software overheads, this reconfiguration time is $11.5 \mu \mathrm{s}$.

2. Determine how precisely the control plane can keep ToR devices synchronized with the OCS. On average, ToRs can be synchronized within $1 \mu \mathrm{s}$. However, due to the non-realtime nature of our Linux-based emulated ToRs, about $1 \%$ of synchronization messages are received at the wrong times, leading to reduced link utilization.

3. Find the overall throughput and circuit utilization delivered by the Mordia OCS. We find that despite the longtailed nature of our synchronization strategy, emulated ToRs can utilize up to $95.4 \%$ of the link's bandwidth.

4. Determine how well TCP performs on the OCS. We find that the emulated ToR can reach $87.9 \%$ of the bandwidth of a comparable EPS for TCP traffic, when disabling the NIC's TSO functionality. The gap between the EPS and OCS is due to the use of commodity hardware and OS in our prototype, and would not be expected in a real deployment.

We evaluate each of these questions below.

\subsection{End-to-end reconfiguration time}

We know from other work [8] that the raw switching speed of the underlying OCS does not determine the end-to-end switching speed, since additional time is required for reinitializing the optics and software overheads. In this section, we empirically measure the OCS switching speed as perceived at a packet level by the devices connected to it. This fundamental switching speed gates the expected performance we expect to see in the remainder of our evaluation.

We first connect 23 emulated ToRs (which we refer to as hosts) to the OCS prototype (with host $i$ connected to port $i$ ). Host 1

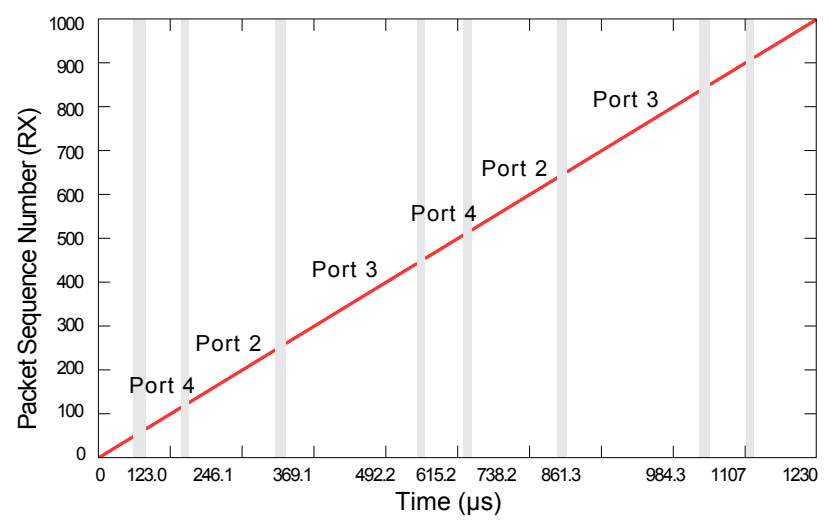

Figure 7: An example of packets sent across a set of variablelength days. Each point represents a single packet, and reconfiguration periods are shown as gray vertical bars.

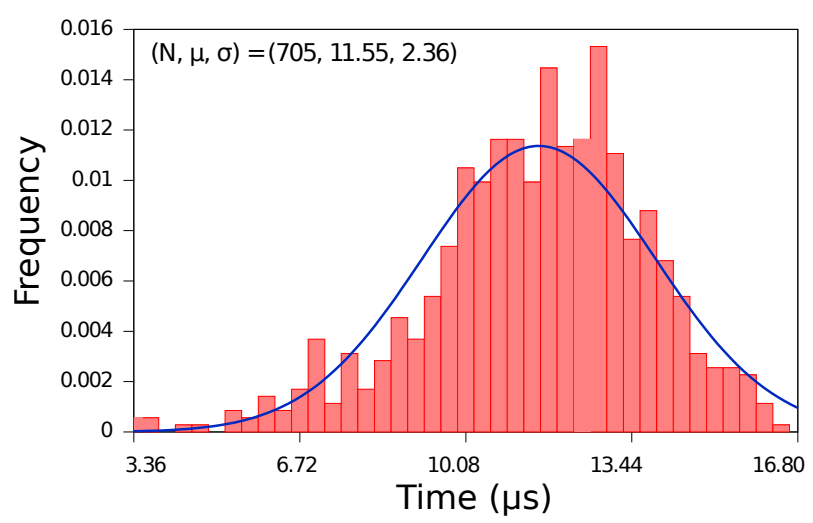

Figure 8: Histogram of the end-to-end OCS reconfiguration time, as seen by connected devices, using 705 samples. A normal curve is fitted to the data.

transmits fixed-size Ethernet frames at line rate, ignoring synchronization packets. Host 1 transmits continuously, even during gaps. Hosts 2 through 23 capture the first 22 octets of each frame using tcpdump. Each frame contains an incrementing sequence number so we can detect loss. After each experiment, we merge the pcap files from each host.

Figure 7 shows an experiment with regular-sized Ethernet frames (1500 bytes) and variable-length slots $(80 \mu \mathrm{s}, 160 \mu \mathrm{s}$, and $240 \mu \mathrm{s})$. The $x$-axis is time and the $y$-axis is packet sequence number. We programmed the OCS with a round-robin schedule. The slot durations vary in size, which means that bandwidth is allocated proportionally to different hosts based on the slot length. We highlight gaps as gray vertical strips, and frames transmitted during gaps are lost. The remaining frames are successfully received. The last packet transmitted during a slot often gets dropped by the receiving NIC because it is cut off in the middle by the OCS.

From a merged pcap trace of approximately one million packets, we extracted 705 gaps in packet transmission. The length of each

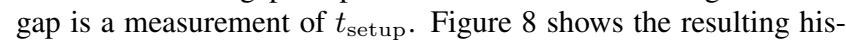
togram. The data fits a normal distribution with a mean of $11.55 \mu \mathrm{s}$ and a standard deviation of $2.36 \mu \mathrm{s}$. Note that this packet capture is collected across several machines, and so some of the variance shown in Figure 8 is due to clock skew across nodes.

With $T=106 \mu$ s and $t_{\text {setup }}=11.5 \mu$ s, the duty cycle is equal 


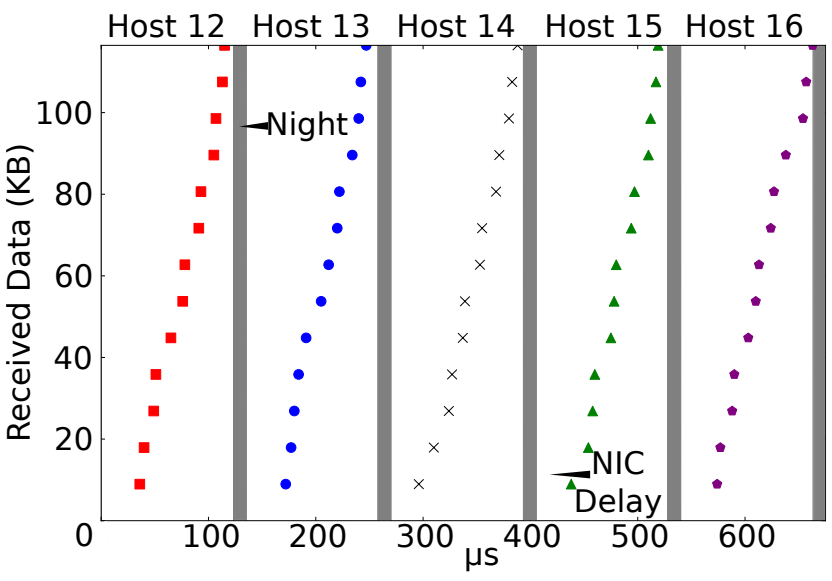

Figure 9: Host 1's Qdisc receiving UDP packets from Hosts 1216 as it cycles through circuits connecting it to 22 other hosts. Each point represents a 9000-byte packet.

to $89.15 \%$. Therefore we expect to have captured $89.15 \%$ of the transmitted Ethernet frames. From 997,917 transmitted packets, we captured 871,731 packets, yielding a measured duty cycle of $87.35 \%$. In other words, there are approximately 18,000 additional missing packets. We attribute these additional missing packets primarily to periods where the sender or receiver was interrupted by the non-real-time Linux kernel.

Summary: These experiments show that the end-to-end reconfiguration latency of the Mordia OCS, including the time to reestablish the link at the optical component level, is on average $11.5 \mu \mathrm{s}$. Further, the FPGA-based scheduler can establish variablelength days and control the OCS with high precision.

\subsection{Emulated ToR software}

The previous section demonstrates that a single host can utilize $87.35 \%$ of a circuit with an $89.15 \%$ duty cycle. However, this measurement does not account for host synchronization at the OCS ports. Figure 9 shows Host 1 receiving 8,966 octet UDP packets from Hosts 12-16 via the Qdisc described in Section 5.2.1 for a day and night of $123.5 \mu \mathrm{s}$ and $11.5 \mu \mathrm{s}$, respectively. The transmission time and sequence number of each packet is determined from a tcpdump trace that runs on only Host 1 .

First, we note that it takes on average $33.2 \mu$ s for the first packet of each circuit to reach Host 1 . The tcpdump trace indicates that it takes less than $3 \mu$ s to process the synchronization frame and transmit the first packet. When sending a 9000-octet frame $(7.2 \mu \mathrm{s})$, the packet spends at least $23 \mu$ s in the NIC before being transmitted. To prevent this "NIC delay" from causing packet loss, the OS Qdisc must stop queuing packets for transmission $23 \mu$ s early. The result is that the Qdisc cannot use $23 \mu$ s of the slot due to the behavior of the underlying NIC hardware. Vattikonda et al. [22] have shown how the use of hardware NIC priority flow control (PFC) pause frames can be used to enable fine-grained scheduling of circuits on (all-electrical) data center networks, and this technique could be applied to Mordia.

Summary: The Linux hosts used as emulated ToRs are able to drain packets into the network during the appropriate "day," which can be as small as $61 \mu \mathrm{s}$. However, jitter in receiving synchronization packets results in a $0.5 \%$ overall loss rate, and there is a $23 \mu$ s delay after each switch reconfiguration before the NIC begins sending packets to the OCS. These overheads and packet loss are specific to our use of commodity hosts as ToRs.

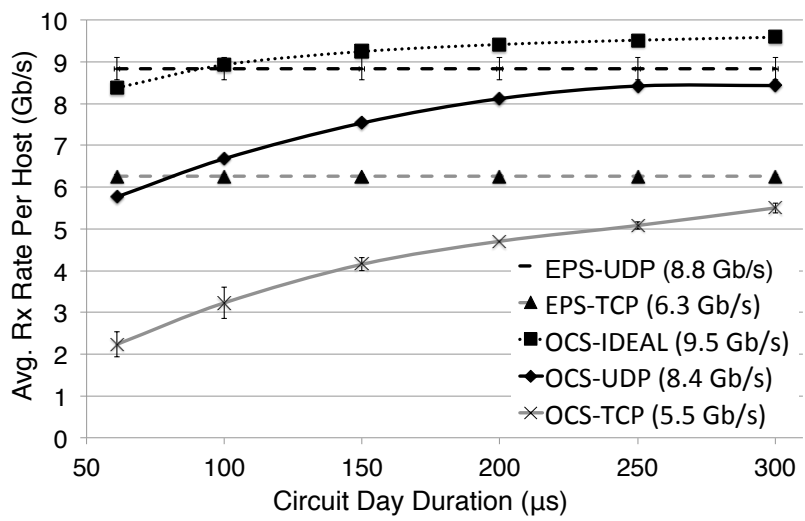

Figure 10: Throughput delivered over the OCS. The fundamental difference between ideal and observed is due to the OCS duty cycle. Further deviations are due to our system artifacts, namely the lack of segmentation offloading in the NIC, NICinduced delay, and synchronization packet jitter. The minimum slot length is $61 \mu \mathrm{s}$. The legend shows the maximum average receive rate for each switch and protocol combination.

\subsection{Throughput}

In the above experiments, we have attempted to characterize individual components of the Mordia OCS. In this section, we turn our attention to analyzing the resulting end-to-end throughput delivered by the entire system.

To begin, we generated all-to-all TCP and UDP traffic between 23 hosts and measured the throughput both over a traditional electrical packet switch (EPS, used as a baseline) as well as our Mordia OCS prototype including emulated ToR switches, shown in Figure 10. The throughput over the EPS serves as an upper bound on the potential performance over the OCS. In addition, throughput over the OCS is fundamentally limited by the OCS duty cycle.

To establish a baseline, we measured the goodput of a single UDP flow transiting the EPS switch (EPS-UDP) to be $9.81 \mathrm{Gbps}$. We then found that as the number of hosts increases from 2 to 23 , the average rate degrades to $8.83 \mathrm{Gbps}$, which we attribute to the kernel and NIC. The EPS supports a single TCP flow's goodput of $8.69 \mathrm{Gbps}$, which is within $1.6 \%$ of UDP traffic. However, this throughout relies on TCP segmentation offloading (TSO) support in the NIC, which is incompatible with our Mordia kernel module. 2 On an all-electrical packet network, all-to-all TCP bandwidth across 23 hosts without TSO support was found to be $6.26 \mathrm{Gbps}$ (EPS-TCP), which we use as an upper bound on the performance we expect to see over the OCS. With NIC firmware access, we could eliminate this reduction in bandwidth by having the TCP offload engine not send packets during the circuit night time.

Figure 10 shows the raw bandwidth available to each host (calculated as the duty cycle) from the OCS as OCS-IDEAL. It is important to remember that this line does not account for the $23.5 \mu \mathrm{s}$ NIC delay which reduces measured duty cycle further. For the experiments, we varied the OCS slot duration between 61-300 $\mu \mathrm{s}$ to observe the effect of different duty cycles (due to the programming time of our WSSes, the smallest slot duration we support is $61 \mu \mathrm{s}$ ). The OCS's UDP throughput (OCS-UDP) ranges from

\footnotetext{
${ }^{2}$ The happens because, when circuits are reconfigured, any packets in flight are 'runted' by the link going down, and we lose control over the transmission of packets when relying on TSO. Consequently, Mordia requires disabling TSO support.
} 


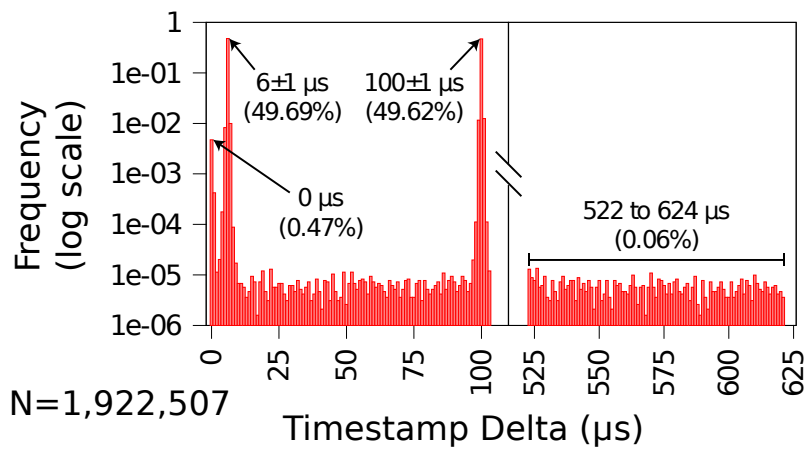

Figure 11: Synchronization jitter as seen by the OS in our Linux-based emulated ToR switches.

5.73-8.43 Gbps, or within $4.6 \%$ of EPS-UDP. The major reasons for the discrepancy are duty cycle, NIC delay, the OS's delay in handling a softirq, and synchronization jitter (see discussion below).

TCP throughput on the OCS (OCS-TCP) ranges from 2.23 to $5.50 \mathrm{Gbps}$, or within $12.1 \%$ of EPS-TCP for circuit day durations. TCP throughput suffers from all of the issues of UDP throughput, as well as two additional ones. First, TCP traffic cannot use TSO to offload, and so the TCP/IP stack becomes CPU-bound handling the required 506 connections. Second, the observed $0.5 \%$ loss rate invokes congestion control, which decreases throughput. However, TCP does show an upward trend in bandwidth with increasing duty cycle.

We suspect that a reason for the reduction in throughput seen in Mordia is due to the "long tailed" behavior of the synchronization aspect of its control plane. To test this, we next examine synchronization jitter. Synchronization packets are generated by the FPGA to minimize latency and jitter, but the OS can add jitter when receiving packets and scheduling softirqs. To measure this jitter, we set the day and night to $106 \mu$ s and $6 \mu$ s, respectively, and capture 1,922,507 synchronization packets across 3 random hosts. We compute the difference in timestamps between each packet and expect to see packets arriving with timestamp deltas of either $6 \pm 1 \mu \mathrm{s}$ or $100 \pm 1 \mu \mathrm{s}$. We found that $99.31 \%$ of synchronization packets arrive at their expected times, $0.47 \%$ of packets arrive with timestamp deltas of zero, and $0.06 \%$ packets arrive with timestamp deltas between $522 \mu$ s and $624 \mu$ s (see Figure 11). The remaining $0.16 \%$ of packets arrive between 7-99 $\mu$ s. We also point out that the $0.53 \%$ of synchronization packets that arrive at unexpected times is very close to our measured loss rate. Our attempts to detect bad synchronization events in the Qdisc did not change the loss rate measurably. Firmware changes in the NIC could be used to entirely avoid the need for these synchronization packets by directly measuring the link up/down events.

Summary: Despite the non-realtime behavior inherent in emulating ToRs with commodity PCs, we are able to achieve $95.4 \%$ of the bandwidth of a comparable EPS with UDP traffic, and $87.9 \%$ of an EPS, sending non-TSO TCP traffic. We are encouraged by these results, which we consider to be lower bounds of what would be possible with more precise control over the ToR.

\section{SCALABILITY}

Supporting large-scale data centers requires an OCS that can scale to many ports. We briefly consider these scalability implications.
WDM: The Mordia prototype we built uses a single ring with 24 wavelength channels in the C-band to create a $24 \times 24$-port OCS. Since the C-band contains 44 DWDM channels, it is straightforward to scale the prototype to 44 ports. Increasing the number of wavelengths on a single ring beyond 44 is more difficult. Mordia happens to rely on $100 \mathrm{GHz}$ spacing, but we could have used $50 \mathrm{GHz}, 25 \mathrm{GHz}$, or even $12.5 \mathrm{GHz}$ spacing. Each smaller increment doubles the number of channels. SFP+ modules with lasers on the $50 \mathrm{GHz}$ grid are commercially available, meaning that it is straightforward to scale to 88 ports. However, the technology to support $10 \mathrm{G}, 40 \mathrm{G}$, and $100 \mathrm{G}$ Ethernet over narrower DWDM channels might not yet be commercially available or might be cost prohibitive in a data center environment. An alternative could be to keep the $100 \mathrm{GHz}$ spacing but to extend into the L-band. This extension would allow a doubling of the number of channels, but would make amplification more difficult. Thus the use of WDM provides a small level of scalability up to a couple of hundred ports.

Bandwidth: As data center network link speeds increase over time, e.g., to 40, 100, and $400 \mathrm{Gbps}$, the Mordia data plane need not change, as it is agnostic to the transmission rate. However, the control plane must increase in speed. Most relevant for Mordia's scalability is not the aggregate per-host bandwidth, but the underlying line rate. We believe that on a five-year time horizon, the fastest line rates will be $25-28$ Gbps. All higher rates will be achieved by aggregating multiple such lanes. For instance, 40 Gbps Ethernet is in fact four lanes of $10 \mathrm{Gbps}$ and the two $100 \mathrm{Gbps}$ standards are either $10 \times 10 \mathrm{Gbps}$ or $4 \times 25 \mathrm{Gbps}$. The emerging $400 \mathrm{Gbps}$ will likely be $16 \times 25 \mathrm{Gbps}$. This aggregation means that the worst-case scaling performance of Mordia is $2.5 \times$ up to $400 \mathrm{Gbps}$. Furthermore, the duty cycle overhead is only a function of the underlying line rate, not the aggregate link rate (e.g., 100 or $400 \mathrm{Gbps}$ ).

Multiple-ring scaling: Another way to scale beyond 88 ports is to use multiple stacked rings, with each ring reusing the same wavelength channels, as shown in Figure 12. For example, an $8 \times 8$ ring-selection OCS would allow the construction of a $8 \times 88=$ 704-port OCS. It is important that all inputs assigned to the same wavelength channel be connected to the same ring-selection OCS, or else there could be a collision within a particular ring. The ringselection OCS is only used for the input ports; the output ports directly connect to the individual rings. One downside of a stacked ring architecture is the longer "week" lengths. Thus for low-latency applications, a packet-switched network is still required.

While the single-ring architecture is fully non-blocking, the stackedring architecture is blocking, meaning that not all input-output port mappings are possible. Fundamentally the challenge comes from reusing a finite number of wavelength channels across a larger number of switch ports. One possible solution to this problem is to introduce another degree of freedom by using tunable lasers that can transmit on any wavelength channel rather than on a specific channel. This freedom restores the fully non-blocking property of the OCS at the cost of additional optical and algorithmic complexity. In terms of scaling to higher port counts, we acknowledge that our proposed approach will not directly apply to networks with clusters larger than 704 ToRs. However, assuming 40 servers/ToR, this constant still scales to 25 thousand servers in a single cluster.

Integrated OCS switching: Finally, it is possible to build an integrated scale-out OCS by interconnecting smaller OCS switches in a multi-stage topology on a single board, using waveguides instead of discrete fibers. This approach greatly reduces loss, since the couplers used to connect the switch to the fibers can be a significant source of loss. Multi-stage, integrated OCSes have been built [3], but rely on slower 3D-MEMS technology. 
Input Ports

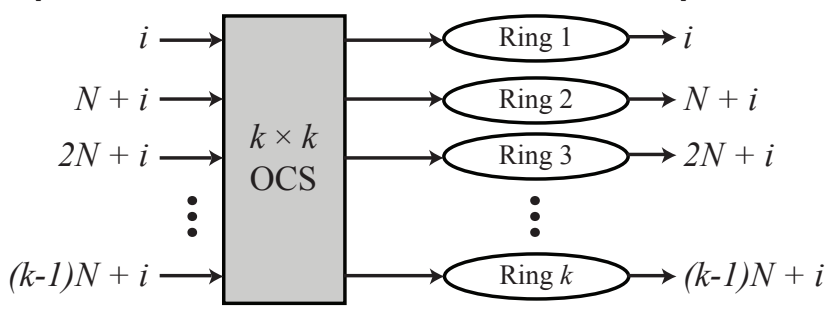

Figure 12: Multiple independent rings can be stacked to increase the total port count. Each of the $k$ rings has $N$ ports. Every input port $j N+i$, where $j \in\{0 . . k-1\}$ and $i \in\{1 . . N\}$, is bundled together into a $k \times k$ ring-selection OCS before being sent to its default ring. This approach allows an input normally destined for one ring to arrive at a different ring.

\section{RELATED WORK}

Optical switching technologies: Realistic optical switches that can be used in practice require a limited overall insertion loss and crosstalk, and must also be compatible with commercial fiber optic transceivers. Subject to these constraints, the performance of a switch is characterized by the switch speed and port count. Optical switches based on electro-optic modulation or semiconductor amplification can provide nanosecond switching speeds, but intrinsic crosstalk and insertion loss limit their port count. Analog (3D) MEMs beam steering switches can have high port counts (e.g., 1000 [4]), but are limited in switching speed on the order of milliseconds. Digital MEMs tilt mirror devices are a "middle-ground". They have a lower port count than analog MEMs switches, but have a switching speed on the order of a microsecond [9] and a sufficiently low insertion loss to permit constructing larger port-count OCSes by composition.

"Hotspot Schedulers": Mordia is complementary to work such as Helios [8], c-Through [25], Flyways [13], and OSA [6], which explored the potential of deploying optical circuit switch technology in a data center environment. Such systems to date have all been examples of hotspot schedulers. A hotspot scheduler observes network traffic over time, detects hotspots, and then changes the network topology (e.g., optically [6, 8, 25] or wirelessly [13]) such that more network capacity is allocated to traffic matrix hotspots and overall throughput is maximized. Rather than pursing such a reactive policy, Mordia instead chooses a proactive scheduling approach in which multiple scheduling decisions are amortized over a single pass through the control plane.

Optical Burst Switching: Optical Burst Switching [17,21] is a research area exploring alternate ways of scheduling optical links through the Internet. Previous and current techniques require the optical circuits to be setup manually on human timescales. The result is low link utilization. OBS introduces statistical multiplexing where a queue of packets with the same source and destination are assembled into a burst (a much larger packet) and sent through the network together. Like OBS, the Mordia architecture has a separate control plane and data plane.

TDMA: Time division multiple access is often used in wireless networks to share the channel capacity among multiple senders and receivers. It is also used by a few wired networks such SONET, ITU-T G.hn "HomeGrid" LANs and "FlexRay" automotive networks. Its applicability to data center packet-switched Ethernet networks was studied in [22], which found that much of the OSbased synchronization jitter can be eliminated by relying on inNIC functionality such as 802.1 Qbb Priority-based pause frames.
By using these pause frames, the NIC can be much more precisely controlled. The approach taken by [22] is not directly applicable to Mordia, since there is no way for a central controller to send pause frames to connected devices when a circuit is not established to that endpoint.

\section{CONCLUSIONS}

In this paper, we have presented the design and implementation of the Mordia OCS architecture, and have evaluated it on a 24-port prototype. A key contribution of this work is a control plane that supports an end-to-end reconfiguration time 2-3 orders of magnitude smaller than previous approaches based on a novel circuit scheduling approach called Traffic Matrix Scheduling. While Mordia is only one piece in a larger effort, we are encouraged by this initial experience building an operational hardware/software network that supports microsecond switching.

\section{ACKNOWLEDGMENTS}

This work is primarily supported by the National Science Foundation CIAN ERC (EEC-0812072) and a Google Focused Research Award. Additional funding was provided by Ericsson, Microsoft, and the Multiscale Systems Center, one of six research centers funded under the Focus Center Research Program (FCRP), a Semiconductor Research Corporation program. We would like to thank our shepherd Arvind Krishnamurthy, and Alex Snoeren and Geoff Voelker for providing invaluable feedback to this work.

\section{REFERENCES}

[1] M. Al-Fares, A. Loukissas, and A. Vahdat. A Scalable, Commodity, Data Center Network Architecture. In Proceedings of ACM SIGCOMM, Aug. 2008.

[2] M. Al-Fares, S. Radhakrishnan, B. Raghavan, N. Huang, and A. Vahdat. Hedera: Dynamic Flow Scheduling for Data Center Networks. In Proceedings of 7th USENIX NSDI, Apr. 2010.

[3] W. Anderson, J. Jackel, G.-K. Chang, H. Dai, W. Xin, M. Goodman, C. Allyn, M. Alvarez, O. Clarke, A. Gottlieb, F. Kleytman, J. Morreale, V. Nichols, A. Tzathas, R. Vora, L. Mercer, H. Dardy, E. Renaud, L. Williard, J. Perreault, R. McFarland, and T. Gibbons. The MONET Project-A Final Report. IEEE Journal of Lightwave Technology, 18(12):1988-2009, Dec. 2000.

[4] D. Beaver, S. Kumar, H. C. Li, J. Sobel, and P. Vajgel. Finding a needle in Haystack: Facebook's photo storage. In Proceedings of 9th USENIX OSDI, Oct. 2010.

[5] G. Birkhoff. Tres Observaciones Sobre el Algebra Lineal. Univ. Nac. Tucumán Rev. Ser. A, 5:147-151, 1946.

[6] K. Chen, A. Singla, A. Singh, K. Ramachandran, L. Xu, Y. Zhang, and X. Wen. OSA: An Optical Switching Architecture for Data Center Networks and Unprecedented Flexibility. In Proceedings of 9th USENIX NSDI, Apr. 2012.

[7] N. Farrington, G. Porter, Y. Fainman, G. Papen, and A. Vahdat. Hunting Mice with Microsecond Circuit Switches. In Proceedings of 11th ACM HotNets, 2012.

[8] N. Farrington, G. Porter, S. Radhakrishnan, H. H. Bazzaz, V. Subramanya, Y. Fainman, G. Papen, and A. Vahdat. Helios: A Hybrid Electrical/Optical Switch Architecture for Modular Data Centers. In Proceedings of ACM SIGCOMM, Aug. 2010.

[9] J. E. Ford, V. A. Aksyuk, D. J. Bishop, and J. A. Walker. Wavelength Add-Drop Switching Using Tilting 
Micromirrors. IEEE Journal of Lightwave Technology, 17:904-911, 1999.

[10] Glimmerglass 80x80 MEMS Switch. http: / / www . glimmerglass.com/products/technology/.

[11] A. Goel, M. Kapralov, and S. Khanna. Perfect Matchings in $O(n \log n)$ Time in Regular Bipartite Graphs. In Proceedings of 42nd ACM STOC, June 2010.

[12] Hadoop: Open source implementation of Map Reduce. http: //hadoop.apache.org/.

[13] D. Halperin, S. Kandula, J. Padhye, P. Bahl, and D. Wetherall. Augmenting Data Center Networks with Multi-Gigabit Wireless Links. In Proceedings of ACM SIGCOMM, Aug. 2011.

[14] U. Hoelzle and L. A. Barroso. The Datacenter as a Computer: An Introduction to the Design of Warehouse-Scale Machines. Morgan and Claypool Publishers, 2009.

[15] N. McKeown, T. Anderson, H. Balakrishnan, G. Parulkar, L. Peterson, J. Rexford, S. Shenker, and J. Turner. OpenFlow: Enabling Innovation in Campus Networks. ACM Computer Communication Review, 38(2), Apr. 2008.

[16] R. N. Mysore, A. Pamporis, N. Farrington, N. Huang, P. Miri, S. Radhakrishnan, V. Subramanya, and A. Vahdat. PortLand: A Scalable, Fault-Tolerant Layer 2 Data Center Network Fabric. In Proceedings of ACM SIGCOMM, Aug. 2009.

[17] C. Qiao and M. Yoo. Optical Burst Switching (OBS) - A New Paradigm for an Optical Internet. Journal of High Speed Networks, 8(1):69-84, 1999.
[18] R. Sinkhorn. A Relationship Between Arbitrary Positive Matrices and Doubly Stochastic Matrices. The Annals of Mathematical Statistics, 35(2):876-879, 1964.

[19] T. A. Strasser and J. L. Wagener. Wavelength-Selective Switches for ROADM Applications. IEEE Journal of Selected Topics in Quantum Electronics, 16:1150-1157, 2010.

[20] Y. Tamir and G. L. Frazier. High-Performance Multi-Queue Buffers for VLSI Communication Switches. In Proceedings of 15th ACM ISCA, May 1988.

[21] J. S. Turner. Terabit Burst Switching. Journal of High Speed Networks, 8(1):3-16, 1999.

[22] B. C. Vattikonda, G. Porter, A. Vahdat, and A. C. Snoeren. Practical TDMA for Datacenter Ethernet. In Proceedings of ACM EuroSys, Apr. 2012.

[23] J. von Neumann. A certain zero-sum two-person game equivalent to the optimal assignment problem. Contributions to the Theory of Games, 2:5-12, 1953.

[24] M. Walraed-Sullivan, K. Marzullo, and A. Vahdat. Scalability vs. Fault Tolerance in Aspen Trees. Technical Report MSR-TR-2013-21, Microsoft Research, Feb 2013.

[25] G. Wang, D. G. Andersen, M. Kaminsky, K. Papagiannaki, T. S. E. Ng, M. Kozuch, and M. Ryan. c-Through: Part-time Optics in Data Centers. In Proceedings of ACM SIGCOMM, Aug. 2010. 